\title{
Les idées pédagogiques de Soler y Arqués. Leur application dans sa " méthode analytique- synthétique »
}

\section{Denise Fischer}

\section{(2) OpenEdition}

Electronic version

URL: https://journals.openedition.org/dhfles/3057

DOI: $10.4000 /$ dhfles.3057

ISSN: 2221-4038

\section{Publisher}

Société Internationale pour l'Histoire du Français Langue Étrangère ou Seconde

\section{Printed version}

Date of publication: 1 June 1999

Number of pages: p.329-345

ISSN: 0992-7654

\section{Electronic reference}

Denise Fischer, "Les idées pédagogiques de Soler y Arqués. Leur application dans sa « méthode analytique-synthétique »", Documents pour I'histoire du français langue étrangère ou seconde [Online], 23। 1999, Online since 04 July 2015, connection on 10 March 2023. URL: http://journals.openedition.org/ dhfles/3057 ; DOl: https://doi.org/10.4000/dhfles.3057

This text was automatically generated on 10 March 2023.

All rights reserved 


\title{
Les idées pédagogiques de Soler y Arqués. Leur application dans sa « méthode analytique-synthétique »
}

\author{
Denise Fischer
}

1 0. Carlos Soler y Arqués (1836-1896), natif de la province de Gerona, étudie à Vich, puis à Saragosse et commence à enseigner comme professeur agrégé au lycée de Huesca en 1862. Il est nommé en 1870 au lycée de Badajoz. Il donne ensuite des cours d'anglais et de français à l'Ecole Centrale des Arts et Métiers et termine sa carrière à l'Institut Cardenal Cisneros de Madrid. Parallèlement à l'enseignement, Soler se consacre aux études historiques et philologiques et reçoit différents prix pour des Mémoires publiés par l'Académie des Sciences Morales et Politiques, ou l'Académie d'Histoire, dont il est membre ou correspondant. Il collabore à diverses revues, écrit un roman, des tableaux de genre, des scènes de mœurs et publie des traductions de l'italien et du portugais. En 1866 paraît son Curso de lengua francesa, puis ses Lecciones de lengua francesa divididas en dos cursos $\left(1884,5^{e}\right.$ éd.) qui connurent un succès certain puisqu'en 1889 apparaissait déjà la $15^{\mathrm{e}}$ édition, suivie d'autres (1895 et la dernière, semble--t-il, en 1924) ${ }^{1}$. Divers écrits reflètent ses préoccupations pédagogiques : Las reformas y la segunda enseñanza. Consideraciones sobre problemas, necesidades y desaciertos, publié en 1891 et, de la même année, La segunda enseñanza y las lenguas vivas.

\section{Contexte pédagogique dans lequel Soler a élaboré ses Leçons}

2 Dans l'introduction de son ouvrage, Soler cite plusieurs auteurs de manuels ou de traités de pédagogie qui ont tenté de réformer renseignement traditionnel des langues en proposant une méthode innovatrice à l'époque. Il s'agit de Ahn, Ollendorff, Robertson, Hamilton, Longchamp et Carderera. 
3 1.1. Le Dr. Ahn commençait son ouvrage par une exposition de ses principes et de sa méthode:

Apprenez une langue étrangère comme vous avez appris votre langue maternelle : voilà en peu de mots la méthode que j'ai suivie dans ce petit ouvrage. C'est la marche que suit la nature elle-même, c'est celle que nous indique la mère qui parle à son enfant, qui lui répète mille fois les mêmes mots, les combine insensiblement et parvient ainsi à lui faire parler la même langue qu'elle parle. Apprendre ainsi n'est plus une étude, c'est un amusement. (Ahn, 1863 : introd.)-

4 Puis il ajoutait qu'il avait introduit quelques règles, intercalées dans ses leçons, afin d'en faciliter l'étude à l'élève qui aurait appris sa langue de façon plus théorique.

5 Cette méthode, destinée aux étudiants en allemand, a connu un grand succès et s'est élargie à d'autres langues : portugais, anglais, français. Près d'un siècle après sa publication elle était encore utilisée, révisée et adaptée pour les Espagnols voulant apprendre le français par Eugenio de Ochoa, puis au début du $\mathrm{XX}^{\mathrm{e}}$ siècle par Atalo Castans.

6 Son succès était dû à la simplicité de ses phrases, à un vocabulaire à complexité progressive et à une répétition fréquente des mots qui revenaient de leçon en leçon, mais combinés de façon différente, se gravant ainsi dans la mémoire de sorte que l'élève, ayant peu d'effort à faire à chaque leçon, arrivait à une connaissance graduelle de la langue. Grâce à cette méthode progressive « hasta las inteligencias mas débiles alcanzan el conocimiento práctico-teórico del idioma con un esfuerzo muy pequeño ${ }^{2}$ » dit Atalo Castans dans son prologue (Castans, 1909 : III). Les mots clés qu'a retenus Soler de cette méthode sont " la connaissance pratique-théorique ", mettant la pratique avant la théorie à l'instar de ce qui se passe dans la langue maternelle.

7 1.2. Ollendorff insistait, lui aussi, dans sa méthode, sur la marche progressive de ses leçons :

De la phrase la plus simple de toutes à la période toute entière, chaque leçon se rattache à la précédente par un mot ou un principe de grammaire dont l'élève sent déjà d'avance le besoin, voit la place et désire la possession, ce qui, excitant sans cesse la curiosité, ajoute encore un vif intérêt à l'étude (Ollendorff, 1853 : V, VI).

8 Nous savons la fortune qu'a connue la méthode ollendorff, appliquée à l'anglais, à l'allemand, au russe et adaptée au français pour les étudiants espagnols par Eduardo Benot qui, terminant la présentation du livre, écrit

Si se sigue este método, sin acortarlo por ningun estilo, y sin omitir ni saltar las preguntas que se hallan en la Clave, aun cuando a veces parezean repetidas, ni los ejercicios que se marcan en la misma, garantizo formalmente, que no habrá discípulo alguno, siempre que no sea imbécil ó idiota, que deje de aprender bien el francés (Benot, $1858: 18)^{3}$.

9 1.3. Théodore Robertson (pseudonyme de Lafforgue) fait paraître en 1837 la première édition de son Cours pratique, analytique, théorique et synthétique de langue anglaise qui connaîtra de multiples rééditions tout au long de la deuxième moitié du XIX ${ }^{\mathrm{e}}$ siècle et qu'imiteront nombre de pédagogues. Soler s'est largement inspiré de la méthode de Robertson - qu'il prend d'ailleurs pour un auteur anglais - tout en critiquant sa façon de présenter la théorie de la langue.

10 Robertson remarque que l'enseignement de ses prédécesseurs était morcelé et isolait complètement la partie de vocabulaire de celle de la grammaire et des idiotismes. Ces trois parties constitutives d'une langue semblaient avoir, entre elles, des cloisons étanches. La base de l'enseignement, pour beaucoup, était constituée par 
l'apprentissage de la grammaire, alors que le vocabulaire était délaissé au profit de la « théorie ». Les manuels antérieurs commençaient tous par des exposés grammaticaux, c'est--à-dire par des abstractions et des généralités, arides et rébarbatives pour les étudiants. Or toute langue est composée de mots et c'est l'étude des mots qui devrait constituer la base de l'enseignement.

Aujourd'hui - déclare Robertson (1875:9) - et j'ai l'orgueil de croire que j'y ai contribué pour quelque chose, on comprend que le fait doit toujours précéder la règle et lui servir de point d'appui.

11 Donc la première phase sera l'apprentissage de mots et de phrases, d'où les règles seront déduites plus tard. Cette étude évitera à l'étudiant l'ennui d'aller consulter le dictionnaire où les mots sont, dit Robertson, relégués " comme dans une fosse commune » (Robertson, $1875: 2$ ). L'étudiant doit alors « les y déterrer avec des peines inouïes et une énorme perte de temps. Cette exhumation est tellement rebutante que l'étudiant n'y recourt qu'à la dernière extrémité et qu'il se garde bien de la pousser au delà des nécessités du moment [...] Le premier problème à résoudre a donc été celui-ci: Faire apprendre les mots sans dictionnaire et par là, réduire à peu près des sept huitièmes le temps de l'étude.»

Quels mots seront étudiés ? Uniquement ceux de la langue usuelle, c'est-à-dire quelques trente mille, ce qui peut sembler un effort de mémoire énorme, mais, tout compte fait, ce chiffre se trouve réduit car on peut lui ôter tous les mots qui présentent une ressemblance avec la langue maternelle, ou bien avec le latin et tous les mots qui sont dérivés d'une même famille. Nous allons retrouver chez Soler, non seulement la même préoccupation pour l'étude des mots, mais aussi un intérêt spécial, dans toutes ses leçons, pour la dérivation, les emprunts, les préfixes et suffixes, etc., qu'il analyse dans la dernière partie de chaque leçon intitulée Estudio filológico de las palabras.

Comment seront étudiés les mots? Après avoir fait tout un travail préalable d'analyse qui consiste en une statistique de la langue, un recueil de racines, une classification des dérivés selon les préfixes et les suffixes, l'indication de la prononciation des mots, la mise en exemples de la grammaire, un ensemble d'idiotismes, Roberston élabore les leçons de son manuel qui comprennent chacune trois parties : la partie pratique, l'analyse et théorie, l'application de la théorie ou exercices.

14 La partie pratique se présente sous une forme narrative : un petit récit familier et amène à la portée de tous les élèves. En effet, Robertson remarque que s'il est difficile de retenir des mots de vocabulaire isolés d'un contexte, ces mêmes mots se graveront beaucoup plus vite dans la mémoire de l'apprenant s'ils sont combinés entre eux, formant des phrases logiques dans un texte court :

Mais si une phrase complète offre plus de prise à la mémoire qu'une série de mots isolés, il est logique d'en conclure, à plus forte raison, - et l'expérience le prouve, qu'un récit suivi s'apprend et se retient beaucoup plus facilement qu'une série de phrases incohérentes. (Robertson, $1875: 11$ ).

Le déroulement de la leçon se fera selon le schéma suivant ;

Partie pratique :

Lecture réitérée à haute voix du texte, avec copie du texte et de sa traduction en regard. Exercice de reconstitution du texte en cachant, tantôt la partie de droite, tantôt celle de gauche.

18 Questions posées sur le texte dans la langue maternelle, auxquelles l'élève doit répondre dans la langue étudiée. 
dans ses Morceaux littéraires (p. 411-415), il critique lui aussi les abus de la grammaire et du dictionnaire, " les deux piliers qui, flanqués de la version, supportaient autrefois tout l'édifice de l'enseignement. » (Soler 1887 : 412). La grammaire est repoussée car rebutante, abstraite et synthétique. Or « l'homme s'instruit par l'observation, l'analyse et l'expérimentation. » (id. : 413). Quant aux dictionnaires, ils ne tiennent pas compte, dit Longchamp, de la formation et dérivation des mots -ce qui pourrait aider l'élève* Grammaire et dictionnaire ne doivent donc être utilisés qu'avec modération.

Carderera, cité amplement dans la $15^{\mathrm{e}}$ édition de Soler, pédagogue de la langue espagnole, remarque que le mal de l'enseignement vient des méthodes et des livres. Il faut avant tout familiariser les enfants avec la pratique, et ensuite viendra la grammaire. Dans ce qu'il appelle les « nouvelles grammaires ou grammaires pédagogiques ", la théorie est subordonnée à la pratique. Au lieu de charger la mémoire de règles et définitions incompréhensibles pour un enfant, on lui donne une série d'exercices gradués logiquement. Nous retrouvons les idées, nouvelles à l'époque, que les « gramáticas ordinarias no sirven para la enseñanza, y no solo esto, sino que son perjudiciales [...] El niño habla por imitación y practica las leyes de la gramática sin conocerlas. Los ejercicios permitirán deducir las prinicipales reglas y definiciones de la gramática. ${ }^{5}$ " (Carderera, $1866: 258$ ). Au chapitre XIV de son livre, consacré à la pédagogie du castillan dans des régions d'Espagne où l'on parle une autre langue, 
Carderera observe que les langues étrangères peuvent s'apprendre de deux façons : celle de la traduction, ou bien celle que la mère utilise avec ses enfants. «El primero da resultados lentos e incompletos; el segundo, mas natural y sencillo, es también mas

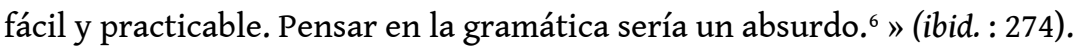

\section{Les idées pédagogiques de Soler}

Ahn, Ollendorff et Hamilton, malgré le renouveau proposé dans leurs méthodes, sont cependant critiqués par Soler qui croit insuffisante la « routine systématique » pour l'étude des langues. Par ailleurs, Robertson, dont il partage les idées, dissémine, dit-il, sa théorie en règles grammaticales sans lien entre elles ${ }^{7}$. Que propose donc Soler?

Il constate que l'enseignement des langues donne peu de résultats pratiques. Quelles en sont les causes?

Tout d'abord le professorat suit dans son enseignement la solution de la facilité, c'est-àdire la routine classique héritée de tout un passé de pédagogues. En quoi consiste cette routine ? En premier lieu en règles de grammaire qui, si elles servent pour se perfectionner dans l'étude d'une langue étrangère, sont arides et parfaitement inutiles pour les élèves qui ignorent les premiers éléments de cette langue. Or « sabemos que la gramática no enseña ni a hablar ni a escribir ${ }^{8} "($ Soler, $1887: 4$ ). Les exemples choisis dans les manuels sont stériles, les thèmes sont oiseux pour ceux qui n'ont pas de connaissances préalables du français. L'étude de la grammaire peut limer, polir, mais il faut un matériel préalable susceptible d'être poli.

31 D'autre part, le but du professeur n'est pas de former des académiciens ni des hommes de lettres, « se trata sencillamente de comunicar un conocimiento generalizado, de dar una práctica útil y bastante para satisfacer las varias necesidades de la vida moderna. ${ }^{9}$ " (ibid.). La grammaire n'est pas une fin en soi-même, elle n'est qu'un moyen parmi tant d'autres et ce moyen peut être facilement remplacé. On peut en effet savoir beaucoup de grammaire et être incapable de parler et d'écrire, et inversement : ignorer les règles de grammaire et être à même de parler. La grammaire, pour Soler, est une série de règles théoriques et de définitions qui perdent leur sens en étant fragmentées. De toute manière, elles dépassent l'entendement d'un enfant. Ce qu'il faut faire, c'est introduire l'enfant dans la pensée même. D'abord le langage, ensuite viendra la grammaire.

32 Le type d'exercices que préconise Soler doit être pratique. Il faut à tout prix éviter la routine afin de faire fonctionner l'intelligence et la réflexion de l'élève. Cette idée sera appliquée à tout moment dans son livre : les exercices après la lecture du texte proposé sont basés sur le réemploi de tout le vocabulaire et des expressions, mais dans des phrases différentes, J'élève n'ayant jamais à répéter textuellement la lecture, mais à modifier sa structure dans une exploitation nouvelle.

Soler remarque une profusion de manuels de français qui sont, dit-il, identiques car copiés les uns des autres, avec quelques suppressions, quelques ajouts, mais, pour ce qui est du fond, rien ne change, la méthode employée ne se questionne pas : la théorie passe toujours avant la pratique.

Or la synthèse n'est pas un procédé naturel. Par contre l'analyse, c'est-à-dire l'observation, est un des plus puissants moyens pour l'homme de s'instruire. Soler résume sa méthode par une formule : " D'abord la pratique, l'étude et la théorie ensuite. » Il présentera donc d'abord les matériaux de la langue : mots, constructions et 
idiotismes, puis les règles qui expliquent d'une façonclaire etsimple le mécanisme dulangage. Il explique ainsi leplan suivi : d'abord lapratique par de brefsrécits, des anecdotes, un vocabulaire statistiquement usuel, une grammaire en exemplesavec une étude philologiquedes mots (leur formation, une comparaison, deleurs, racines-, préfixes etsuffixes....) C'est, lui semble-t-il, la façon la plus rationnelle de présenter l'étude d'une langue étrangère-. D'où le sous-titrede son manuel: Método analíticosintético.

\section{L'application des idées de Soler dans son manuel}

Soler commenceson ouvragepar une Introduction dans; laquelle ildéfinit les différents termes grammaticaux, puis par desPréliminaires; qui consistent en uneétude de la phonologie. Il. s'agit, du strict indispensable, sans entrer dans le détail des prononciationsparticulières qui ennuieraient l'élève, et que- d'ailleurs les Français euxmêmes n'introduisentpas. dans leurs manuels pour enfants. Cetteinitiation, à la prononciation française, accompagnée d'exercicesde lecture, luipermettra, d'éliminer de ses leçons les références phonétiques. En effet, à la différence de Robertson, nous ne trouvons pas, aucours; des leçons, d'explications phonétiques supplémentairesou de transcriptions.

Après laphonologie et avant lapremière leçon, l'édition de 1889 propose» dans une Nota Previa, un découpage temporel des leçons : trois jours pour voir successivement l'historiette quisert de thème, lesexercices pratiques de phraséologie etd'analyse basés sur la lecture de la leçon et celle des leçons antérieures qui ont été rédigées dans un ordre progressif, ensuite l'étude de la comparaison et de la formation des mots, et la partie grammaticale indispensable. Les élèves devront apprendre par cœur ce qui est écrit en caractères d'imprimerie normaux, ce qui est écrit en caractères plus petitsétant réservés pour l'étude en classe.

\section{Structure du livre et des leçons ;}

Le manuel comprend 6 petits chapitres dans la partie préliminaire de phonologie et 40 leçons divisées en deux cours de longueur égale et intitulées Lecciones de construcción y lexigrafía. Nous prendrons comme exemple les premières leçons pour nous former une idée de la méthode employée par Soler.

La structure en est toujours la même. Soler présente en premier lieu une lecture assez courte - pour le début - avec sa traduction interlinéaire. Les mots de cette traduction sont peu à peu supprimés au fur et à mesure de leur apparition dans le texte, ainsi dans la première phrase :

Une bonne femme avait une poule, bonne pondeuse

Una buena mujer tenía gallina, ponedora

« une » et « bonne » n'apparaissent plus traduits, l'élève étant censé faire l'effort de mémoire pour retenir tous les mots vus. A cette lecture et traduction linéaire, suit une traduction « correcte » où le texte est présenté dans un bon espagnol et non plus dans un mot à mot. L'élève est ainsi à même de constater les transformations nécessaires lors du passage de la langue étrangère à sa langue maternelle. A partir de la leçon 31 , il n'y a plus de traduction interlinéaire, Soler se contente d'indiquer un numéro entre parenthèses qui renvoie au bas de la page à la traduction du mot en espagnol, et à la fin 
du texte il signale : "Faites la traduction littérale. » Ensuite vient la traduction « correcte ».

La troisième étape, qui s'appelle conversation est divisée en deux colonnes : à gauche des questions en espagnol sur le texte qui vient d'être lu et traduit, et à droite les réponses en français, que l'élève trouvera dans la lecture. Il s'agit donc de localiser le passage auquel se réfère la question, et de répéter les mots textuellement. Cela pour vérifier la compréhension et pour exercer la mémoire par la répétition. C'est le même principe qu'avait appliqué Robertson, et comme lui, Soler passe à la vingtième leçon à la question posée directement dans la langue d'apprentissage et non plus dans la langue maternelle de l'élève. Cela, dit-il, stimule l'élève, lui donnant la conscience de ce qu'il a acquis en si peu de temps.

Le quatrième exercice, intitulé Phraséologie - tout comme dans le manuel de Robertson est disposé aussi en deux colonnes : celle de gauche en espagnol pour la traduire en français, et celle de droite en français, pour traduire en espagnol. Cet exercice est plus élaboré que le précédent et demande de la part de l'élève un effort plus grand. En effet, il ne s'agit plus de répéter des mots isolés ou des bribes de phrases, mais il faut maintenant agencer entre eux divers éléments épars dans le texte. En combinant les mots déjà connus dans de nouvelles phrases ayant un sens complet, on évite ainsi la répétition monotone et ennuyeuse pour l'élève et les mots se gravent dans la mémoire. Il est à supposer que cet exercice de phraséologie se faisait dans les deux sens (thème et version), l'élève cachant d'abord une colonne, puis l'autre colonne et passant à la phase suivante quand le vocabulaire était mémorisé.

Ensuite nous arrivons à l'analyse, c'est-à-dire à l'explication et exploitation grammaticale et lexicale du texte présenté. On en signale les particularités morphologiques (pour la lère leçon : les articles indéfinis, les verbes qui sont apparus), on fait remarquer le pluriel de quelques noms, et à l'occasion de ces mots on énonce la règle de formation du pluriel, on note l'apostrophe de pronoms (l'), le féminin de quelques adjectifs (bonne, grasse), et du point de vue lexical, on élargit le vocabulaire étudié en donnant des dérivés (de poule : poulet, poulette, poularde ; de jour : journal, journalier, journaliste, journée, journellement...) ou en révélant la polysémie de quelques mots (bonne, bête : adjectifs ou noms). Toutes ces explications sont données en espagnol.

44 Vient ensuite l'Etude philologique des mots, en espagnol également. Dans cette partie, Soler étudie l'étymologie, les emprunts aux diverses langues, les ressemblances de radicaux, les mots composés, les suffixes néo-latins, les préfixes grecs et latins, mots à aphérèse, circonlocutions, etc. Dans l'édition de 1887, seul le $1^{\text {er }}$ cours présentait cette Etude philologique. Dans celle de 1889 , Soler l'a étendue à son $2^{\mathrm{e}}$ cours. Il $y$.étudie .principalement la formation des mots et la dérivation.

45 La dernière phase de la leçon est constituée par la Phraséologie qui, dans le $1^{\text {er }}$ cours, se réduit à la conjugaison des verbes. Soler commence par les auxiliaires (deux premières leçons); ensuite il passe à l'emploi de chacun de ces auxiliaires et quels verbes régissent l'un et l'autre aux temps composés (L. 3). Suivent les autres conjugaisons à tous les temps et à tous les modes... Ce n'est que dans la $2^{\text {e }}$ partie du livre ( $2^{\circ}$ Curso) qu'apparaissent l'étude de l'article, les degrés de l'adjectif, possessifs, démonstratifs, indéfinis, pronoms personnels, relatifs, la formation du pluriel des noms, les adverbes, prépositions, conjonctions. On s'attendrait en fait à ce que certains points grammaticaux soient exposés avant le $2^{\mathrm{e}}$ cours car il est évident que l'élève a dû les 
utiliser dès la leçon I du premier cours. Dans la partie de la leçon intitulée Analyse, Soler explique d'ailleurs les principales nouveautés grammaticales. L'exposé grammaticalest donc un traité récapitulatif de ce qui a déjà été vu analytiquement. C'est: la synthèse de toutes les explications précédentes et de toutes, les observations qu'a pu faire l'élève au cours de sa $\mathrm{I}^{\mathrm{e}}$ année de français. Tous les points grammaticaux sont ainsi analysés dans leur contexte et font, dans le $2^{\mathrm{e}}$ cours, l'objet d'un exposé théorique et synthétique. Soler remédie, de cette façon, à la dissémination de la théorie en règles grammaticales sans lien entre elles, qu'il critiquait chez Robertson. ${ }^{10}$

Après la partie grammaticale, à partir de la leçon 4 , Soler propose parfois un thème : des phrases en espagnol à traduire en français pour appliquer la règle étudiée. Ce type d'exercices est généralisé à partir de la leçon 21 ( $2^{\mathrm{e}}$ cours).

\section{Critique de cette méthode et conclusion}

Le vocabulaire employé par Soler dans ses Leçons est-il bien choisi ? La notion de " fonctionnel » n'existe pas encore à l'époque. Robertson indiquait qu'il avait fait une statistique exacte de la langue. Il est probable que Soler aussi, avant d'écrire ses petits récits, s'est pose la question de savoir quel type de vocabulaire venait en priorité. Or, dès la $\mathrm{I}^{\mathrm{e}}$ leçon, nous trouvons les mots suivants : pondre, pondeuse, engraisser, poule et ses dérivés ; $2^{\mathrm{e}}$ leçon : coq ; $3^{e}$ leçon : la perdrix, le perdreau, couver, mots qui ne sont pas d'une fréquence telle que leur apprentissage soit indispensable à un débutant.

D'autre part les premiers textes ne présentent aucun temps présent, mais des passés simples, imparfaits (et même conditionnels dans le $1^{\text {er }}$ texte). Le présent étant le temps le plus employé, et le passé simple réservé au récit écrit, les conversations menées entre professeur et étudiant dans la partie de Phraséologie apparaissent un peu factices. Par expérience nous savons aussi que la notion imparfait / passé simple est, pour les élèves, assez difficile à saisir. Or les lectures jusqu'à la leçon 4 , où apparaît un seul présent, ne contiennent que ces deux temps verbaux.

49 Le verbe est étudié dans ses moindres détails dans le $1^{\text {er }}$ cours, car Soler pense qu'il est d'une importance capitale. Mais est-il vraiment indispensable de l'étudier à tous ses temps et à toutes ses formes dès la $\mathrm{I}^{\mathrm{e}}$ leçon ? Dans la leçon 3 , on explique à l'élève quels sont les verbes qui se conjuguent avec être et quels sont ceux qui se conjuguent avec avoir. Or le passé composé n'est pas encore apparu dans les lectures, puisque c'est le passé simple qui est employé. Soler diffère ici de Carderera en ce que celui-ci pense qu'on ne doit pas commencer à conjuguer par les verbes auxiliaires car ce sont les plus abstraits de tous, ils sont irréguliers et ne peuvent être utilisés comme type. « Tampoco conviene estudiar todos los modos y tiempos seguidos, porque no se forma idea exacta de ellos y ofrecen grande complication para los niños. ${ }^{11}$ " (Carderera, $1866: 269$ ).

Par ailleurs, la partie Etude philologique des mots, appelée dans l'édition de 1889 Etude comparative des mots, est peut-être un peu trop complète pour un élève de secondaire à qui est destinée cette méthode. Elle reflète cependant une préoccupation linguistique nouvelle dans ce type de manuel, le désir de faire un ouvrage scientifique et moderne.

Un des grands attraits de ce manuel, c'est l'acquisition progressive et méthodique du vocabulaire. Les questions sur le texte, auxquelles l'élève ne répondra que s'il a lu attentivement le récit plusieurs fois, et l'a analysé, l'obligeront à un effort de mémoire presque imperceptible. Ensuite le réemploi des expressions et du vocabulaire dans 
d'autres phrases lui permettra un élargissement de ses connaissances en combinant le déjà étudié dans les leçons précédentes au tout récemment vu dans la leçon du jour. Cette méthode d'analyse qui fait ensuite la synthèse des acquis, puis énonce des règles générales connaîtra un énorme succès, la preuve en est les dix rééditions du livre en cinq ans (1884-1889).

Soler se place, avec ses Leçons, dans une lignée de pédagogues qui préconisent la pratique avant la théorie. Radonvilliers, Du Marsais - pour n'en citer que quelques-uns $\mathbf{s}^{12}$ - avaient déjà introduit la méthode de double traduction qui sera appliquée au XIX ${ }^{\mathrm{e}}$ siècle non plus pour le latin, mais pour les langues vivantes.

\section{BIBLIOGRAPHY}

AHN, Hans Franz $\left(1863^{17}\right)$, Nouvelle Méthode pratique et facile pour apprendre la langue allemande, 1er cours, Leipzig, F. A. Brockhaus, Paris, Lib. A. Franck.

BENOT, Eduardo $\left(1858^{5}\right)$, Ollendorff reformado. Gramática francesa y método para aprenderla, $5^{\mathrm{a}}$ ed. corregida nuevamente, Cádiz, « Revista Médica».

BESSE, Henri (1991), « Les techniques de traduction dans l'étude des langues au XVIII ${ }^{\mathrm{e}}$ siècle » in Documents pour l'histoire du français langue étrangère ou seconde, 8, 77-95.

- (1996), «Traduction interlinéaire et enseignement des langues » in Documents pour l'histoire du français langue étrangère ou seconde, 18., 293-312.

CARDERERA Y POTO, Mariano (186633), Principios de educacin y métodos de enseñanza, Madrid, Imp. del Colegio de Sordo mudos y de Ciegos.

CASTANS, Atalo (1909), Método de Ahn, Primer curso de francés seguido de un compendio de gramática francesa, revisado por..., Madrid, Bailly Baillère.

DELABORDE $\left(1855^{2}\right)$, Novísimo arte teórico, práctico, analítico y sintético de la lengua francesa para usa de los españoles, Madrid, Imp. de J. de M. Gonzalez.

HAMiLton, H. (?), Premières notions de langue anglaise avec la prononciation écrite, Méthode Robertson, Paris, chez l'Auteur, Lib. française et anglaise de J. H. Truchy, Leroy frères successeurs.

OLLENDORFF, H. G. $\left(1853^{2}\right)$, Nouvelle méthode pour apprendre à lire, à écrire et à parler une langue en six mois. Appliquée à l'anglais, $2^{\mathrm{e}}$ éd. revue, corrigée et augmentée, Paris, chez l'Auteur. ROBERTSON, Théodore (1875), Nouveau cours pratique, analytique, théorique et synthétique de langue anglaise en trois parties, Paris, E. Derache, $15^{\mathrm{e}}$ éd.

SOLER Y ARQUÉs, Carlos $\left(1887^{\circ}\right)$, Lecciones de lengua francesa bajo un plan sencillo y agradable. Método analítico-sintético, Madrid, Tip. de Manuel Gines Hernández.

$1889^{15}$, id., corregida y aumentada, Madrid, Vda de Hernando.

1898, Programa para los exámenes de Lengua Francesa arreglado a las lecciones de D...., Madrid, Imp. de Hemando ycia. 


\section{NOTES}

1. Nous nous appuierons pour cette étude sur la $9^{\mathrm{e}}$ éd. de 1887 et sur la $15^{\mathrm{e}}$ de 1889 .

2. « même les intelligences les plus faibles atteignent à une connaissance pratique-théorique de la langue avec un moindre effort. »

3. «Si l'on suit cette méthode, sans l'abréger ni omettre ou sauter les questions qui se trouvent dans la Clé, même si quelquefois elles semblent se répéter, ni les exercices qui y sont marqués, je garantis formellement qu'il n'y aura aucun élève, à moins qu'il ne soit imbécile ou idiot, qui n'apprenne pas bien le français. »

4. Soler retiendra cette idée que l'étude des racines et des familles de mots peut soulager la mémoire de l'étudiant et il l'appliquera dans ses Leçons,

5. «grammaires ordinaires ne servent pas pour l'enseignement, et sont en outre préjudiciables [...] L'enfant parle par imitation et applique les lois de la grammaire sans les connaître. Les exercices permettront de déduire les principales règles et définitions de la grammaire. »

6. «La première donne des résultats lents et incomplets ; la seconde, plus naturelle et simple, est aussi plus facile et pratiquable. Penser à la grammaire serait chose absurde. »

7. «Un práctico autor inglés, Robertson, con quien en esta parte nos conformamos, disemina la teórica en reglas gramaticalmente inconexas. » (Soler, $1887: 7$ ).

8. « nous savons que la grammaire n'enseigne ni à parler ni à écrire. »

9. « il s'agit simplement de communiquer une connaissance généralisée, de donner une pratique utile et suffisante pour satisfaire les différents besoins de la vie moderne.»

10. Robertson avait d'ailleurs prévu cette critique : "On me fera une objection c'est que pour avoir sous les yeux la grammaire complète et dans son ordre régulier, il faut chercher les règles une à une dans les trois volumes du cours, et les transcrire. Je pourrais répondre que ce n'est pas un mal, que c'est même un excellent moyen de retenir ces règles. [...] Mais [...] j'ai fait de l'Index qui termine le $3^{\mathrm{e}}$ et dernier volume, une grammaire alphabétique, où les solutions sont peut-être plus faciles à trouver que dans les grammaires ordinaires. De plus et par surcroît, j'ai mis à la fin de ce premier volume une récapitulation des principes élémentaires, pour les personnes qui n'ont besoin que de- notions générales. » (Robertson» $1875: 16$ ).

11. Il ne convient pas non plus d'étudier tous les modes et les temps les uns après les autres, car on ne s'en fait pas une idée exacte, et ils offrent une grande complication pour les enfants. »

12. Voir les articles de Henri Besse $(1991,1996)$.

\section{AUTHOR}

\section{DENISE FISCHER}

Université Rovira i Virgili, Tarragona 